\title{
FREQUENCY OFFSET ESTIMATION FOR KSP-OFDM
}

\author{
Dieter Van Welden, Heidi Steendam and Marc Moeneclaey \\ Department of Telecommunications and Information Processing, Ghent University \\ Sint-Pietersnieuwstraat 41, 9000 GENT, BELGIUM \\ E-mail: \{dmvwelde,hs,mm\}@telin.ugent.be
}

\begin{abstract}
In this paper, we propose two ad hoc frequency offset (FO) estimation algorithms in a known symbol padding (KSP) orthogonal frequency-division multiplexing (OFDM) system, where the guard interval is filled with pilot symbols. Besides those time domain pilot symbols, some additional pilot symbols are transmitted on the pilot carriers. The FO is estimated without any channel knowledge. One algorithm operates in the time domain (TD), while the other one operates in the frequency domain (FD). The interference from the unknown data symbols is much smaller in the FD than in the TD, especially for small FOs. As a result, the FD initialization algorithm results in a lower mean squared error (MSE). Both estimation algorithms reach the BER performance of a receiver with perfect knowledge about the FO.
\end{abstract}

\section{INTRODUCTION}

In recent years it has been shown that Orthogonal Frequency Division Multiplexing (OFDM) [1] is a worthy technique to achieve a high data rate transmission over multipath fading channels. As a result, a number of standards is already based on OFDM systems: xDSL [2], digital audio and video broadcasting [3], [4], wireless LAN [5], [6], etc. To tackle the inter block interference, which is typical for dispersive channels, an OFDM system inserts a guard interval between successively transmitted OFDM blocks. Among the most popular techniques to fill the guard interval are the cyclic prefix (CP) technique and the zero-padding technique ( $\mathrm{ZP})$ (see [7], [8]). In the CP technique, the last samples of each OFDM block are copied and transmitted before the actual OFDM block, whereas the guard interval is left empty in the $\mathrm{ZP}$ technique. For these two techniques, the content of the guard interval is predefined (zeros for the ZP technique and the last samples of the OFDM block, which depend on the unknown data symbols, for the CP technique). As a result, the guard interval is not very useful for channel estimation and sometimes insufficient for synchronization purposes [9]. In this contribution a third guard interval technique is considered: known symbol padding (KSP) [9], where the guard interval is filled with pilot symbols. Since we have full control about which pilot symbols are transmitted during the guard interval, we can optimize the pilot sequence so that it can be useful for synchronization tasks. In [10] for example, a time delay estimation algorithm is proposed for KSP-OFDM that outperforms existing time delay estimation algorithms for CP-OFDM.

One of the problems a receiver has to deal with in an OFDM system, is the presence of a frequency mismatch between the oscillators in the transmitter and the receiver. A frequency offset (FO) causes inter carrier interference and attenuates the useful signal [11]. When an FO (even of the order of a fraction of the carrier spacing) is not compensated, the bit error rate (BER) performance can be severely degraded. To our knowledge, no FO estimation algorithms for a KSP-OFDM system have been proposed in the literature. Therefore we present in this contribution two ad hoc FO estimation algorithms: one that operates in the time domain (TD) and one that operates in the frequency domain (FD). Both algorithms exploit the presence of the pilot symbols on the pilot carriers (FD pilots) and in the guard interval (TD pilots) in the received signal that corresponds to two or more consecutively transmitted OFDM blocks.

Both estimation algorithms show an error floor for the mean squared error (MSE), which is caused by the interference from the unknown data symbols. The error floor can be lowered by increasing the number of considered OFDM blocks to estimate the FO . For the bit error rate (BER), the performance of a receiver with perfect knowledge about the FO but with estimated channel is reached by both estimation algorithms.

\section{SYSTEM MODEL}

We consider a KSP-OFDM system with $N$ carriers and a guard interval consisting of $\nu$ samples. The transmitted symbols on the carriers during the $i$-th OFDM block are denoted as $\mathbf{a}_{i}=\left(a_{i}(0), \ldots, a_{i}(N-1)\right)^{T}$. During each OFDM block, $M-\nu$ FD pilot symbols, denoted as $\mathbf{b}_{c}=\left(b_{c}(0), \ldots, b_{c}(M-\nu-1)\right)^{T}$, and $N-M+\nu$ data symbols, denoted as $\mathbf{a}_{d}^{(i)}=$ $\left(a_{d}^{(i)}(0), \ldots, a_{d}^{(i)}(N-M+\nu-1)\right)^{T}$, are transmitted. The set of carrier indices is divided in a subset $S_{p}=$ $\left\{\alpha_{0}, \ldots, \alpha_{M-\nu-1}\right\}$, consisting of pilot carriers, and a subset $S_{d}=\left\{\beta_{0}, \ldots, \beta_{N+\nu-M-1}\right\}$, which contains the 
a) transmitter

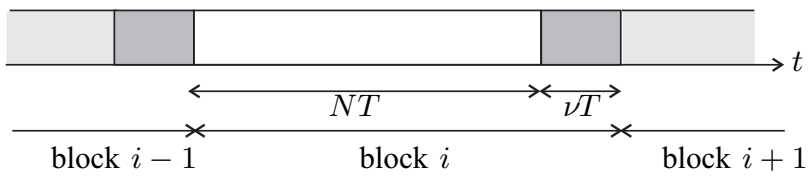

b) receiver

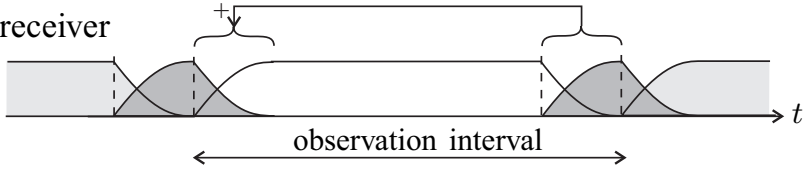

Fig. 1. Time-domain signal of KSP-OFDM a) transmitted signal b) received signal and observation interval

data carriers. The guard interval consists of $\nu$ (TD) pilot symbols denoted as $\mathbf{b}_{g}=\left(b_{g}(0), \ldots, b_{g}(\nu-1)\right)^{T}$, so per OFDM block a total number of $M$ pilot symbols is transmitted. The $N+\nu$ transmitted time-domain samples corresponding to the $i$-th block are obtained by feeding $\mathbf{a}_{i}$ to the inverse Fast Fourier Transform (IFFT) and by inserting the guard interval after the $N$ IFFT outputs :

$$
\mathbf{s}_{i}=\sqrt{\frac{N}{N+\nu}}\left(\begin{array}{c}
\mathbf{F}^{H} \mathbf{a}_{i} \\
\mathbf{b}_{g}
\end{array}\right),
$$

where $\mathbf{F}$ is the $N \times N$ FFT matrix with $\mathbf{F}_{k, l}=$ $(1 / \sqrt{N}) e^{-j 2 \pi \frac{k l}{N}}$. We assume that the data symbols are independent and identically distributed (i.i.d.) and the energy per symbol is equal to $E_{s}$. Further we assume that $E\left[\left|b_{c}(n)\right|^{2}\right]=E\left[\left|b_{g}(k)\right|^{2}\right]=E_{s}$. The normalization factor $\sqrt{N /(N+\nu)}$ in (1) is necessary to make sure that $E\left[\left|\mathbf{s}_{i}\right|^{2}\right]=N E_{s}$. The vector $\mathbf{s}_{i}$ can be expressed as the sum of a vector $\mathbf{s}_{p}$, which collects the contributions from both the pilot symbols in time and frequency domain, and a vector $\mathbf{s}_{d}^{(i)}$, which consists of the contributions from the unknown data symbols:

$$
\mathbf{s}_{i}=\mathbf{s}_{p}+\mathbf{s}_{d}^{(i)} .
$$

The OFDM blocks are transmitted over a frequency selective channel with impulse response $\mathbf{h}=(h(0), \ldots, h(L-1))^{T}$ where $L$ is the number of samples in the channel impulse response. To avoid inter block interference, the length of the guard interval can not be smaller than the duration of the channel impulse response, i.e. $\nu \geq L-1$. The $N+\nu$ received time-domain samples corresponding to the observation interval shown in figure $1 . b$ are given by

$$
\mathbf{r}_{i}=e^{j 2 \pi \frac{i(N+\nu)}{N} \epsilon} \mathbf{E}(\epsilon) \tilde{\mathbf{H}} \mathbf{s}_{i}+\mathbf{w}_{i} .
$$

where $\epsilon$ denotes the unknown FO between the transmitter and receiver oscillators expressed as a fraction of the inter carrier spacing. We assume that an initial estimation of the
FO already has been performed so that $|\epsilon|<0.5 N /(N+\nu)$. Typically, a coarse FO estimate is obtained using a suitable preamble which is transmitted before the actual OFDM data blocks (see for example the IEEE 802.11 preamble structure [6, sec. 17.3.3]). The $(N+\nu) \times(N+\nu)$ channel matrix $\tilde{\mathbf{H}}$ is given by

$$
(\tilde{\mathbf{H}})_{k, k^{\prime}}=h\left(\left|k-k^{\prime}\right|_{N+\nu}\right)
$$

where $|x|_{K}$ is the notation of the modulo- $K$ operation which gives the remainder of the division of $x$ by $K$. The diagonal matrix $\mathbf{E}$ is defined as: $(\mathbf{E}(\epsilon))_{k, k}=e^{j 2 \pi \frac{k}{N} \epsilon}$ and the vector $\mathbf{w}_{i}$ represents the white Gaussian noise added by the channel. The real and imaginary parts of the components of $\mathbf{w}_{i}$ are i.i.d. with zero mean, and variance $N_{0} / 2$.

The data detection process in a KSP-OFDM system consists of a sequence of operations that have to be carried out. First, the FO estimate is used to compensate the FO in the time domain. Then the contributions from the guard interval samples are removed from the received signal samples using the channel estimate. The resulting received signal is similar to a received signal from a ZP-OFDM system. The last $\nu$ samples of the considered observation interval in figure 1 are added to the first $\nu$ samples, to restore the orthogonality between the carriers (see [7]). After the removal of the guard interval samples, the resulting first $N$ samples are applied to an FFT to transform the frequency selective channel in $N$ parallel flat fading channels. Finally per carrier singletap equalization, for which the channel impulse response $\mathbf{h}$ is needed, and data detection are performed. Note that the receiver has to estimate $\epsilon$ and $\mathbf{h}$ first, to be able to perform data detection. The two proposed FO estimation algorithms to estimate $\epsilon$ are introduced in the next section. For the channel estimation we consider the algorithm proposed in [12].

\section{FREQUENCY OFFSET ESTIMATION}

Both estimation algorithms need the received signal samples of $K(\geq 2)$ consecutively transmitted OFDM blocks, to start from. We denote them as $\mathbf{r}_{i}, \ldots, \mathbf{r}_{i+K-1}$, which are given by (3):

$$
\begin{aligned}
\mathbf{r}_{i}= & e^{j 2 \pi \frac{i(N+\nu)}{N} \epsilon} \mathbf{E}(\epsilon) \tilde{\mathbf{H}}\left(\mathbf{s}_{p}+\mathbf{s}_{d}^{(i)}\right)+\mathbf{w}_{i} \\
\mathbf{r}_{i+K-1}= & e^{j 2 \pi \frac{(i+K-1)(N+\nu)}{N} \epsilon} \mathbf{E}(\epsilon) \tilde{\mathbf{H}}\left(\mathbf{s}_{p}+\mathbf{s}_{d}^{(i+K-1)}\right) \\
& +\mathbf{w}_{i+K-1}
\end{aligned}
$$

The pilot symbol contribution in $\mathbf{r}_{i+k}$ (i.e. the contribution from $\mathbf{s}_{p}$ ), with $k=1, \ldots, K-1$, is equal to the pilot symbol contribution in $\mathbf{r}_{i}$ multiplied by a factor $e^{j 2 \pi \frac{k(N+\nu)}{N} \epsilon}$. We will exploit this fact to estimate the FO $\epsilon$. 


\section{III-A. TD Estimation}

The first estimation algorithm operates in the time domain. When we consider the unknown data symbols as an additional noise term in (4), we can rewrite (4) as

$$
\mathbf{r}_{i+k}=e^{j 2 \pi \frac{(i+k)(N+\nu)}{N} \epsilon} \mathbf{E}(\epsilon) \tilde{\mathbf{H}} \mathbf{s}_{p}+\tilde{\mathbf{w}}_{i+k}
$$

where $\tilde{\mathbf{w}}_{i+k}=\mathbf{w}_{i+k}+e^{j 2 \pi \frac{(i+k)(N+\nu)}{N} \epsilon} \mathbf{E}(\epsilon) \tilde{\mathbf{H}} \mathbf{s}_{d}^{(i+k)}$. We compute the product of the Hermitian transpose of $\mathbf{r}_{i+k}$ with $\mathbf{r}_{i+k+1}$ for $k=0, \ldots, K-2$. The summation of those $K$ products yields a quantity that can be used to estimate $\epsilon$ :

$$
\sum_{k=0}^{K-2} \mathbf{r}_{i+k}^{H} \mathbf{r}_{i+k+1}=(K-1) e^{j 2 \pi \frac{N+\nu}{N} \epsilon}\left|\tilde{\mathbf{H}} \mathbf{s}_{p}\right|^{2}+\sum_{k=0}^{K-2} n_{k}
$$

where $n_{k}$ collects the contributions from $\mathbf{r}_{i+k}^{H} \mathbf{r}_{i+k+1}$ which depend on the noise and the unknown data symbols. The estimate of $\epsilon$ is then given by

$$
\hat{\epsilon}=\frac{1}{2 \pi} \frac{N}{N+\nu} \angle\left\{\sum_{k=0}^{K-2} \mathbf{r}_{i+k}^{H} \mathbf{r}_{i+k+1}\right\} .
$$

The main advantage of this approach is the fact that we do not need to send the received signal through an FFT first.

\section{III-B. FD Estimation}

First we transform the received vectors $\mathbf{r}_{i+k}, k=$ $0, \ldots, K-1$ to the frequency domain. To achieve this, the last $\nu$ samples of each vector are added to the first $\nu$ samples. The first $N$ samples of the resulting vectors are then applied to an FFT. The output of the FFT of OFDM block $i+k$ at carrier $n$ is given by

$$
\begin{aligned}
& Y_{i+k}(n)= \\
& e^{j 2 \pi \frac{(i+k)(N+\nu)}{N} \epsilon}\left(H(\epsilon, n) \sum_{m=0}^{M-\nu-1} b_{c}(m) I_{\alpha_{m}-n}(\epsilon)\right. \\
& \quad+H(\epsilon, n) \sum_{m=0}^{N+\nu-M-1} a_{d}^{(i+k)}(m) I_{\beta_{m}-n}(\epsilon) \\
& \left.\quad+\sum_{l=0}^{L-1} B_{g}(\epsilon, n, l) h(l)\right)+W_{i+k}(n)
\end{aligned}
$$

where $I_{m}(\epsilon), H(\epsilon, n)$ and $B_{g}(\epsilon, n, l)$ are given by

$$
\begin{aligned}
I_{m}(\epsilon)= & \frac{1}{\sqrt{N(N+\nu)}} \frac{1-e^{j 2 \pi(m+\epsilon)}}{1-e^{j 2 \pi \frac{(m+\epsilon)}{N}}} \\
H(\epsilon, n)= & \sum_{l=0}^{L-1} h(l) e^{-j 2 \pi \frac{(n-\epsilon) l}{N}} \\
B_{g}(\epsilon, n, l)= & \frac{1}{\sqrt{N+\nu}}\left(\sum_{k=0}^{l-1} b_{g}(\nu+k-l) e^{j 2 \pi \frac{(\epsilon-n) k}{N}}\right. \\
& \left.+\sum_{k=l}^{\nu-1} b_{g}(k-l) e^{j 2 \pi \frac{(\epsilon-n)(N+k)}{N}}\right)
\end{aligned}
$$

and $\alpha_{m}$ and $\beta_{m}$ are carrier indices belonging to the subsets of carrier indices of pilot carriers and data carriers, respectively. The samples $W_{i+k}(n)$, with $n=0, \ldots, N-1$, are Gaussian noise samples with zero mean and an autocorrelation matrix $\mathbf{R}$ defined as

$$
(\mathbf{R})_{n, n^{\prime}}=N_{0}\left(\delta_{n, n^{\prime}}+\frac{1}{N} \sum_{k=0}^{\nu-1} e^{-j 2 \pi \frac{\left(n-n^{\prime}\right) l}{N}}\right) .
$$

We collect the $M-\nu$ FFT outputs from the $K$ OFDM blocks corresponding to the set $S_{p}$ of pilot carriers in the vectors $\mathbf{y}_{i+k}, k=0, \ldots, K-1$ :

$$
\begin{aligned}
\mathbf{y}_{i+k} & =\left[Y_{i+k}\left(\alpha_{0}\right), \ldots, Y_{i+k}\left(\alpha_{M-\nu-1}\right)\right]^{T} \\
& =e^{j 2 \pi \frac{(i+k)(N+\nu)}{N} \epsilon}\left(\tilde{\mathbf{b}}+\tilde{\mathbf{a}}_{i+k}\right)+\mathbf{W}_{i+k}
\end{aligned}
$$

where $\tilde{\mathbf{b}}$ is the vector that contains the contributions from both time and frequency domain pilots. The vector $\tilde{\mathbf{a}}_{i+k}$ collects the contributions from the unknown data symbols from the $(i+k)$-th OFDM block and $\mathbf{W}_{i+k}$ contains the noise samples at the pilot carrier positions of the $(i+k)$ th block. The summation over $k$ of the multiplications of the Hermitian transpose of $\mathbf{y}_{i+k}$ with $\mathbf{y}_{i+k+1}$ for $k=$ $0, \ldots, K-2$ results in a function which is used to estimate $\epsilon$ :

$$
\sum_{k=0}^{K-2} \mathbf{y}_{i+k}^{H} \mathbf{y}_{i+k+1}=(K-1) e^{j 2 \pi \frac{(N+\nu)}{N} \epsilon}|\tilde{\mathbf{b}}|^{2}+\sum_{k=0}^{K-2} \tilde{n}_{k}
$$

where $\tilde{n}_{k}$ collects the contributions from the unknown data symbols and the noise samples from $\mathbf{y}_{i+k}^{H} \mathbf{y}_{i+k+1}$. The estimate of $\epsilon$ is then given by

$$
\hat{\epsilon}=\frac{1}{2 \pi} \frac{N}{N+\nu} \angle\left\{\sum_{k=0}^{K-2} \mathbf{y}_{i}^{H} \mathbf{y}_{i+1}\right\} .
$$

\section{SIMULATION RESULTS AND DISCUSSION}

In this section, the performance of the proposed frequency estimation algorithms is evaluated. We consider a frequency selective Rayleigh fading channel consisting of $L=50$ channel taps with equal variance. The channel impulse response is normalized: $\sum_{l=0}^{L-1} E\left[|h(l)|^{2}\right]=1$. For the pilots transmitted on the carriers, the comb-type pilot arrangement from [13] is used. The pilot symbols are randomly selected QPSK symbols. The pilot symbols are equally spaced over the pilot carriers: $S_{p}=\{m \lambda \mid m=0, \ldots, M-\nu-1\}$, where $\lambda=$ floor $(N /(M-\nu))$. We consider an OFDM system with $N=1024$ carriers and a guard interval of length $\nu=100$. Besides the 100 pilot symbols in the guard interval, an additional 100 carriers are selected as pilot carriers which means that a total number of $M=200$ pilot symbols are transmitted. The data symbols are QPSK symbols.

First we evaluate the performance of the estimators in terms of the MSE, which is defined as:

$$
M S E=E\left[|\epsilon-\hat{\epsilon}|^{2}\right] \text {. }
$$



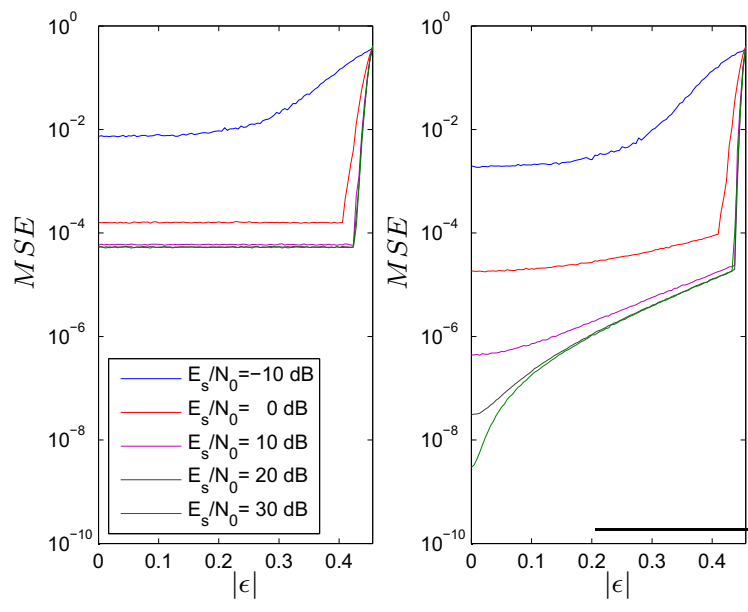

Fig. 2. MSE versus $|\epsilon|$ for TD (left) and FD (right) estimation, $\nu=100, N=1024, M=200, K=10$.

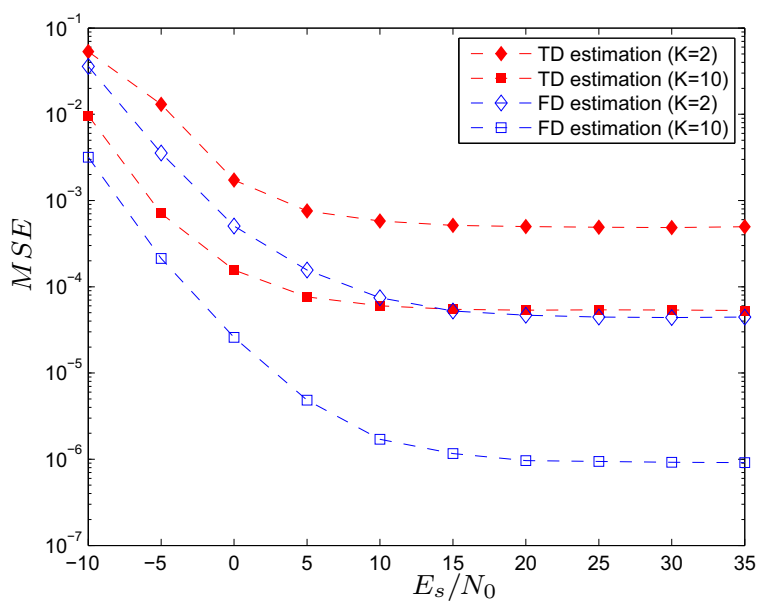

Fig. 3. MSE versus $E_{s} / N_{0}, \nu=100, N=1024, M=200$

Figure 2 shows the MSE of the TD and FD estimation algorithms versus $|\epsilon|$ for different values of $E_{s} / N_{0}$. To obtain the results in figure 2, we have considered $K=10$ received OFDM blocks. We see that both estimators have a bad performance for $|\epsilon|>0.3$. This is due to the discontinuity of the $\angle\{$.$\} function in (8) and (10). Therefore for the$ following simulation results we will assume that $|\epsilon| \leq 0.3$. For the TD estimation we see that the MSE is only weakly dependent on $\epsilon$ and for high values of $E_{s} / N_{0}$ independent of $E_{s} / N_{0}$. The performance of the FD estimator on the contrary, strongly depends on the value of $\epsilon$, especially for high $E_{s} / N_{0}$. For high values of $\epsilon$, the MSE becomes independent of the $E_{s} / N_{0}$. As a result, both estimators will show an error floor for high values of the $E_{s} / N_{0}$ when we plot the MSE versus the $E_{s} / N_{0}$, as can be seen from figure

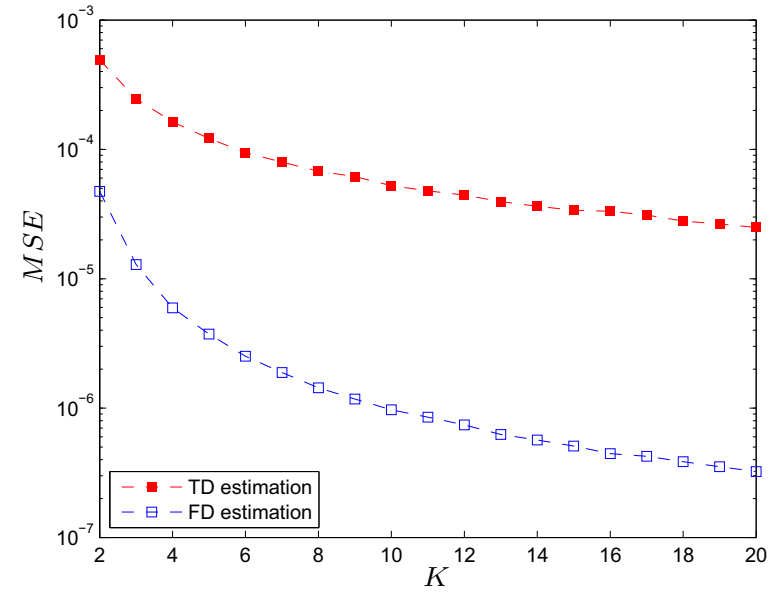

Fig. 4. MSE versus $K, \nu=100, N=1024, M=200$, $E_{s} / N_{0}=20 \mathrm{~dB}$

3. This error floor is caused by the unknown data symbols which interfere with the pilot symbols. We see that the FD estimation obtains the lowest error floor. This behavior is expected because for very small values of $\epsilon$, the inter carrier interference becomes very small. In that case the interference of the unknown data symbols will be much smaller in the FD (and eventually equal to zero when $\epsilon=0$ ), while the interference in the TD is independent of the presence of a FO.

As already can be seen from figure 3, the MSE for both estimation methods also depends on the value of $K$. Figure 4 provides some better insights on the dependence of the MSE on the number of considered OFDM blocks. The considered $E_{s} / N_{0}$ is equal to $20 \mathrm{~dB}$. The results are similar for both proposed algorithms: The MSE decreases when the number of considered OFDM blocks increases because we get a better averaging of the contribution of the unknown data symbols.

Figure 5 shows the BER results. For low to moderate $E_{s} / N_{0}$, the BER of a receiver which applies the TD or FD estimation algorithm for the estimation of the FO, is close to the case when the FO is perfectly known (but with estimated channel). Increasing the number of received OFDM blocks that are used to estimate the FO results in a better BER performance especially for high $E_{s} / N_{0}$ for both estimation algorithms.

\section{CONCLUSION}

In this contribution, we present two ad hoc FO estimation algorithms: TD estimation and FD estimation. Both exploit the presence of the pilot carriers and the pilot symbols in the guard interval to estimate the FO without any knowledge about the channel. The MSE of both estimation algorithms shows an error floor for moderate to high $E_{s} / N_{0}$ but FD 


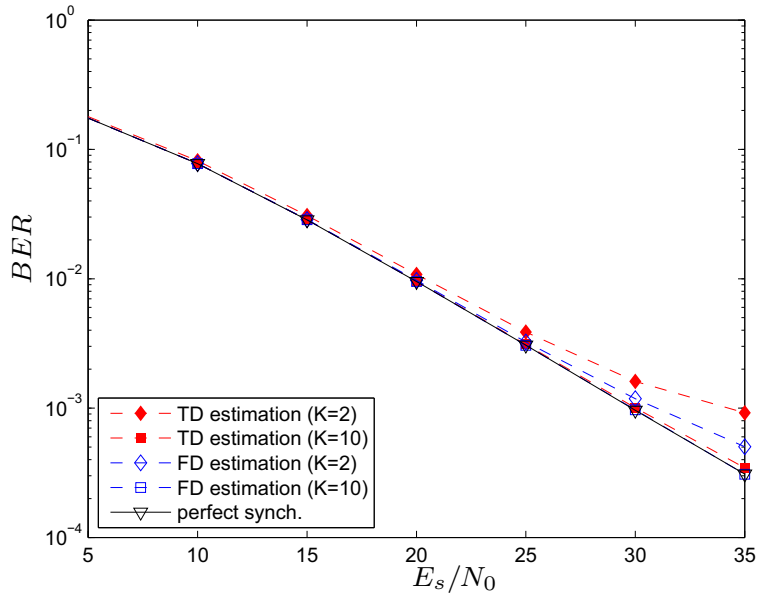

Fig. 5. BER results, $\nu=100, N=1024, M=200$

estimation gives the better performance. The MSE can be decreased by increasing the number of received OFDM blocks to estimate the FO. A receiver with one of the proposed estimation algorithms to estimate the FO, reaches the BER of a receiver with perfect FO knowledge.

The TD estimation method has the advantage that no extra FFT operation is needed, so it has a lower computational complexity.

\section{ACKNOWLEDGMENT}

The authors wish to acknowledge the activity of the Network of Excellence in Wireless COMmunications NEW$\mathrm{COM}++$ of the European Commission (contract n. 216715) that motivated this work.

\section{REFERENCES}

[1] J. A. C. Bingham, "Multicarrier modulation for data transmission, an idea whose time has come," IEEE Communications Magazine, vol. 28, no. 5, pp. pp. 514, May 1990.

[2] European Telecommunications Standards Institute, "Transmission and multiplexing (tm); access transmission systems on metallic access cables; very high speed digital subscriber line (vdsl); part 2: Transceiver specification,” ETSI TS 101 270-2, Feb. 2001.

[3] European Telecommunications Standards Institute, "Digital audio broadcasting (dab); dab to mobile portable and fixed receivers," France, ETS 300 401, Feb. 1995.

[4] European Telecommunications Standards Institute, "Digital video broadcasting (dvb); framing structure, channel coding and modulation for 11/12 ghz satellite services," France, ETS 300 421, Dec. 1994.

[5] R. van Nee, G. Awater, M. Morikura, H. Takanashi, M. Webster, and K. W. Halford, "New high-rate wireless lan standards," IEEE Communications Magazine, vol. 37, no. 12, pp. 82-88, Dec. 1999.

[6] IEEE 802.11, “Wireless lan medium access control (mac) physical layer (phy) specifications, amendment 1: High-speed physical layer in the 5 ghz band," July 1999.

[7] B. Muquet, Z. Wang, G.B. Giannakis, M. de Courville and P. Duhamel, "Cyclic prefixing or zero padding for wireless multicarrier transmissions," IEEE Transactions on Communications, vol. 50, no. 12, pp. 21362148, Dec. 2002.

[8] H. Steendam and M. Moeneclaey, "Different guard interval techniques for ofdm: Performance comparison," in Proc. from 6th International Workshop on MultiCarrier Spread Spectrum (MC-SS'07), Herrsching, Germany, May 2007, pp. 11-24.

[9] L. Deneire, B. Gyselinckx, and M. Engels, "Training sequence versus cyclic prefix - a new look on single carrier communication," IEEE Communications Letters, vol. 5, no. 7, pp. 292-294, July 2001.

[10] D. Van Welden, H. Steendam and M. Moeneclaey, "Time delay estimation for ksp-ofdm systems in multipath fading channels," in Proceedings of the 20th Personal, Indoor and Mobile Radio Communications Symposium, 2009. PIMRC-09, Sept. 2009.

[11] T. Pollet, M. Van Bladel, and M. Moeneclaey, "Ber sensitivity of ofdm systems to carrier frequency offset and wiener phase noise," IEEE Transactions on Communications, vol. 43, no. 234, pp. 191-193, feb/mar/apr 1995.

[12] D. Van Welden, H. Steendam and M. Moeneclaey, "Frequency-domain data-aided channel estimation for ksp-ofdm," in Proc. from 10th International Symposium on Spread Spectrum Techniques and Applications (ISSSTA'08), Bologna, Italy, Aug. 2008.

[13] F. Tufvesson, T. Maseng, "Pilot assisted channel estimation for ofdm in mobile cellular systems," in Proc. of IEEE Veh. Tech. Conf., Phoenix, U.S.A., May 1997, pp. 1639-1643. 